01

\title{
Моделирование оптических концентраторов для модернизированной камеры черенковского гамма-телескопа TAIGA-IACT
}

\author{
(C) А.С. Антонов, А.А. Богданов, А.М. Красильщиков, Е.Е. Холупенко \\ Физико-технический институт им. А.Ф. Иофрфе РАН, \\ 194021 Санкт-Петербург, Россия \\ e-mail: andrey.s.antonov@gmail.com
}

Поступило в Редакцию 19 марта 2021 г.

В окончательной редакции 17 июня 2021 г.

Принято к публикации 18 июня 2021 г.

Выполнено количественное моделирование системы оптических концентраторов на основе шестиугольных конусов Уинстона, предназначенных для камеры регистрации черенковского гамма-телескопа TAIGA-IACT. Получены данные по трансмиссии конусов, приведены распределения интенсивности потока фотонов в плоскости детектора. На основе полученных результатов предложена оптимальная конфигурация оптических концентраторов, учитывающая конструктивные особенности монтировки, зеркала и камеры TAIGA-IACT, a также особенности ее новых детекторных блоков.

Ключевые слова: черенковский гамма-телескоп, TAIGA-IACT, конус Уинстона, численное моделирование.

DOI: 10.21883/JTF.2021.11.51517.67-21

\section{Введение}

В настоящее время черенковские гамма-телескопы являются основным типом астрономических инструментов в диапазоне энергий выше $0.1 \mathrm{TeV}$. Обладая весьма значительной $\left(\sim 10^{4}-10^{5} \mathrm{~m}^{2}\right)$ эффективной площадью детектирования, они позволяют измерять достаточно слабые потоки теравольтного гамма-излучения ряда космических источников (см., например, [1,2]). Такая большая площадь детектирования обусловлена тем, что наблюдение осуществляется не прямым методом, а посредством регистрации черенковского излучения электронов и позитронов широких атмосферных ливней (ШАЛ), инициированных первичными космическими гамма-квантами при взаимодействии с атмосферой Земли. Характерный поперечный размер ШАЛ составляет порядка 200-500 m. Большая площадь детектирования и относительно низкая стоимость черенковских гамма-телескопов обеспечивают настолько значительные конкурентные преимущества, что, по всей видимости, в обозримом будущем они останутся основными инструментами гамма-астрономии в диапазоне энергий выше $0.1 \mathrm{TeV}$. Проекты черенковских телескопов нового (IV) поколения $[3,4]$ находятся в активной фазе, а существующие черенковские телескопы III поколения постоянно модернизируются $[5,6]$.

Начиная с 2019 г. в ФТИ им. А.Ф. Иоффе реализуется проект, направленный на модернизацию камеры черенковского гамма-телескопа TAIGA-IACT (НИИЯФ МГУ, Иркутский университет) [7,8]. Основная цель проекта разработка новых детекторных кластеров для камеры TAIGA-IACT на основе кремниевых фотоумножителей (SiPM), которые позволят повысить эффективность работы этого телескопа путем снижения пороговой энергии наблюдений, увеличения длительности рабочего цикла и т. п. [9-11].

Детекторные камеры черенковских гамма-телескопов обычно состоят из нескольких сотен фотоумножителей $(Ф Э У) ~[12,13]$, каждый из которых оснащается световым концентратором (как правило, конусом Уинстона), выполняющим несколько функций [14,15], как то:

1) переход от размера пикселя, который определяется размером области фокальной плоскости, где формируется изображение черенковской вспышки ШАЛ, и количеством пикселей камеры, к размеру входного окна выбранного фотоэлемента, т. е. фактически осуществление дополнительной концентрации фотонов полезного сигнала;

2) переход от формы пикселя (как правило, шестиугольник), которая должна обеспечивать заполнение детекторной плоскости без пробелов и наложений с целью снижения площади „мертвых“ (не регистрирующих) областей камеры, к форме входного окна выбранного фотоэлемента (в случае традиционного вакуумного ФЭУ, как правило, круг);

3) уменьшение шумового фона фотонов от источников, располагающихся на земной поверхности, и фона фотонов ночного неба, отраженных от земной поверхности.

Данное устройство представляет собой внеосевой параболоид вращения и собирает совокупность лучей, позволяя внеосевым лучам осуществлять неоднократные отражения при прохождении от входной апертуры к выходной.

В настоящее время в камере телескопа TAIGA-IACT используются конусы с окнами в форме правильных шестиугольников, размером выходного окна около $14.8 \mathrm{~mm}$ (диаметр вписанной окружности), что соответствует размеру $15 \mathrm{~mm}$ круглого входного окна ФЭУ ХР1911 [16]. 
План модернизации этой камеры предполагает использование в качестве фотоэлементов сборки из четырех SiPM OnSemi MicroFJ-60035 с входным окном квадратной формы с размером около $12.8 \mathrm{~mm}$ [9]. Такие отличия могут потребовать разработки новых световых концентраторов. Для того чтобы оценить степень необходимости и масштаб изменений конструкции световых концентраторов (конусов Уинстона) для модернизированной камеры TAIGA-IACT по сравнению с используемыми в настоящее время, было проведено предварительное моделирование конусов Уинстона с помощью пакета ZEMAX [17]. В настоящей статье представлены результаты этого моделирования.

\section{1. Постановка задачи}

Основной характеристикой конуса Уинстона является угол обзора $\theta$, равный углу наклона оси параболы к оси конуса (рис. 1). Данная величина, с одной стороны, определяет область пропускания конуса в пространстве углов, а с другой - отношение площадей входного и выходного окна.

В настоящей работе рассмотрена упрощенная (полигональная) конструкция конусов, состоящая из набора параболических поверхностей; таким образом, входное и выходное отверстия конусов представляют собой многоугольники. На рис. 2 показан общий вид опытного образца шестиугольного конуса Уинстона с нанесенной на внутреннюю поверхность отражающей пленкой, изготовленного для новой камеры TAIGA.

Исходя из требований к конструкции камеры регистрации телескопа для численного моделирования, были выбраны шестиугольные конусы Уинстона, которые по своим характеристикам близки к идеальному случаю парабалоида вращения. Расчеты, приведенные в работе [19], показали, что трансмиссия шестиугольного конуса Уинстона практически не отличается от его осесимметричного аналога при углах конуса около 30 градусов и более. Под трансмиссией конуса подразумевается выраженное в процентах отношение количества фотонов, прошедших через конус, к количеству фотонов, поступивших на входное окно конуса.

Численное моделирование было проведено в программном комплексе ZEMAX, предназначенном для расчетов оптических систем и оптоэлектронных приборов. Для расчета задач геометрической оптики этот пакет использует трассировку лучей по методу Монте Карло, суть которого заключается в отслеживании траектории лучей и расчета взаимодействий с лежащими на траекториях объектами. Моделирование конусов Уинстона проводилось в режиме непоследовательной трассировки лучей, который подразумевает, что лучи могут попадать на любую поверхность любого объекта в произвольной последовательности, могут попадать на один и тот же объект несколько раз или вообще не попадать на него. В процессе моделирования производился расчет

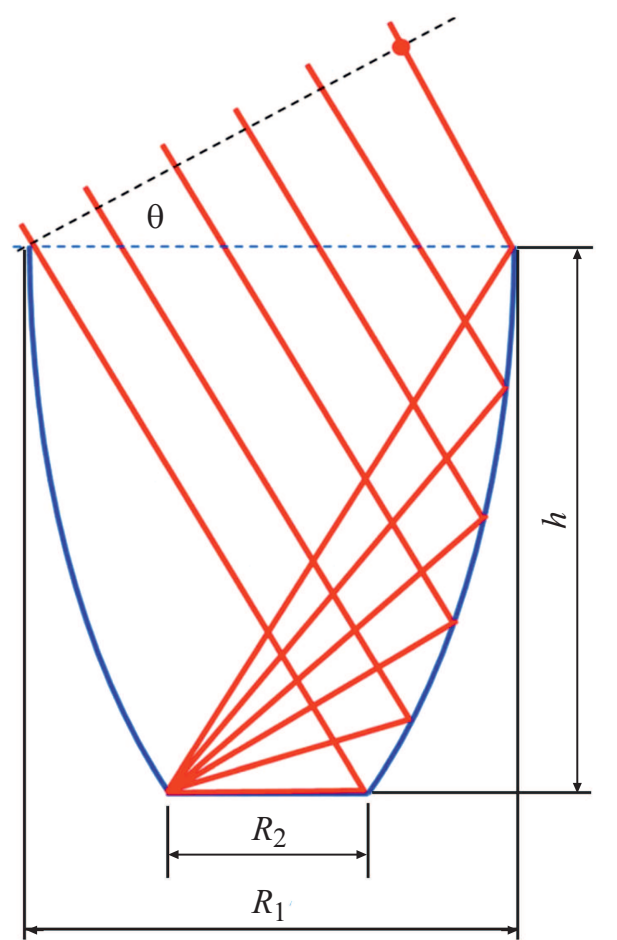

$a$

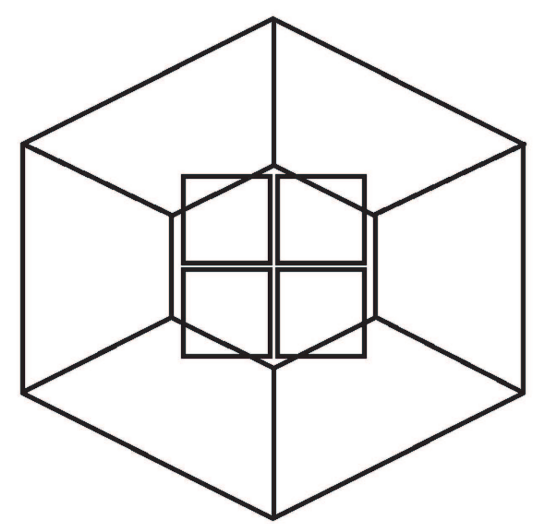

$b$

Рис. 1. Схема конуса Уинстона; $a-$ вид сбоку [18], $b-$ вид сверху. Квадратами указано положение детекторов.

траекторий движения световых лучей в системе оптического концентратора с учетом отражения от внутренних поверхностей конуса. Перед проведением численного моделирования для системы оптических концентраторов было составлено техническое задание, согласно которому коэффициент отражения внутренней поверхности конусов Уинстона должен быть не менее 0.95 в диапазоне длин волн $300-700 \mathrm{~nm}$. Именно эта величина использовалась в моделировании.

Численная модель включала параболическое зеркало телескопа, одиночный конус Уинстона, а также набор из 4 детекторов, расположенных у выходного окна конуса (рис. 2). Геометрические параметры зеркала телескопа были приняты следующими: радиус зеркала - $2.16 \mathrm{~m}$, фокусное расстояние - $4.75 \mathrm{~m}$. Геометрические параметры конусов приведены в табл. 1. Каждый из 4 


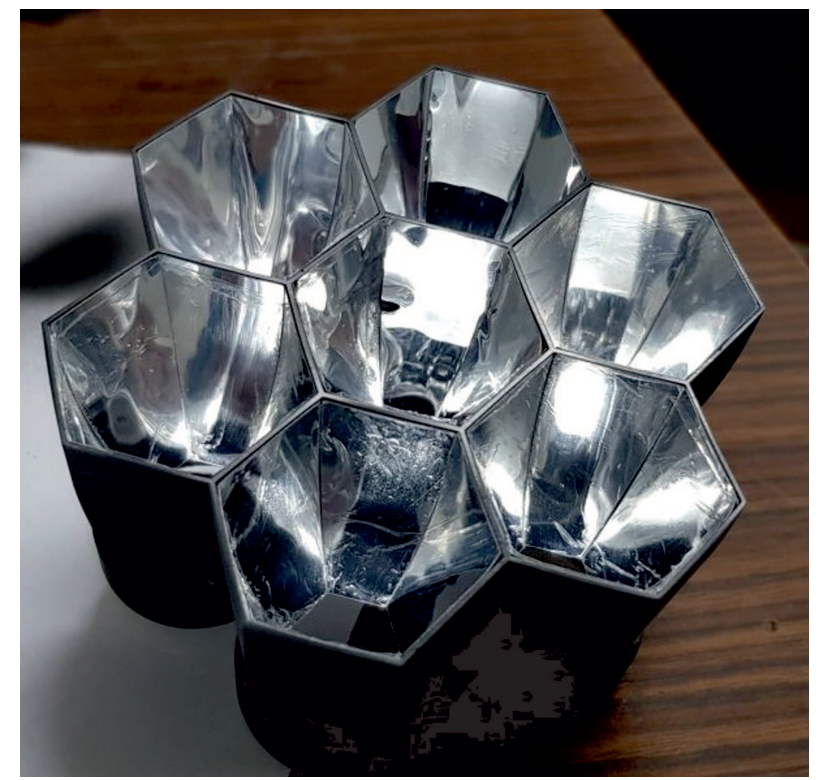

Рис. 2. Опытные образцы шестиугольных конусов Уинстона с нанесенной на внутреннюю поверхность отражающей пленкой.

Таблица 1. Геометрические параметры конусов Уинстона с различными углами $\theta$

\begin{tabular}{c|c|c|c}
\hline Параметры & $\theta=26.56^{\circ}$ & $\theta=30.00^{\circ}$ & $\theta=35.00^{\circ}$ \\
\hline$R_{1}, \mathrm{~mm}$ & 15 & 15 & 15 \\
$R_{2}, \mathrm{~mm}$ & 6.50 & 7.50 & 8.60 \\
$h, \mathrm{~mm}$ & 44.77 & 38.97 & 33.70 \\
Расстояние до & 1 & 1 & 1 \\
детекторов, mm & & &
\end{tabular}

детекторов имеет форму квадрата со стороной $6.13 \mathrm{~mm}$, центры детекторов расположены в углах квадрата со стороной $6.33 \mathrm{~mm}$.

При определении геометрических параметров конуса были использованы следующие соотношения:

$$
\begin{gathered}
\frac{R_{2}}{R_{1}}=\sin \theta, \\
h=\frac{R_{1}+R_{2}}{\operatorname{tg} \theta},
\end{gathered}
$$

где $R_{1}$ и $R_{2}$ - радиусы вписанной окружности для входного и выходного окна соответственно, $\theta-$ угол конуса, $h$ - высота конуса (рис. 1$)$.

\section{2. Результаты численного моделирования}

Были рассмотрены 3 варианта конструкции конусов, отличающиеся углом $\theta$, значение которого бралось равным 26.56, 30 и 35 градусам соответственно. В частности, для случая $\theta=30.00^{\circ}$ отношение площадей вход- ного и выходного окна конуса равно 4. Таким образом, теоретически поток регистрируемых фотонов при использовании конусов Уинстона и заданной площади поверхности детекторов также может быть увеличен в 4 раза. Однако на практике эта величина может быть существенно меньше из-за различного рода потерь фотонов, таких, как потери при отражении от поверхностей конуса, а также потери вследствие неидеальности конуса. Найденная численно функция пропускания для конуса с $\theta=30.00^{\circ}$ приведена на рис. 4. Данная функция представляет собой отношение количества фотонов, прошедших через конус, к количеству фотонов, попавших на входную поверхность конуса при фиксированном угле наклона траектории фотонов к оси конуса $\theta^{\prime}$. При построении данной зависимости производилось численное моделирование 10000000 траекторий фотонов. Полученная функция пропускания, в частности, показывает эффективность подавления шумового фона фотонов (для углов наклона траектории фотона к оси конуса $\left.\theta^{\prime}>\theta=30.00^{\circ}\right)$.

По результатам моделирования был определен угловой размер пикселя системы зеркала телескопа и одиночного конуса, представляющего собой область на небесной сфере в форме шестиугольника с угловым радиусом вписанной окружности $10.8^{\prime}$.

В процессе численного моделирования фотоны стартовали с плоскости 2 , указанной на рис. 3 , в направлении зеркала телескопа с равномерным распределением в пространстве координат внутри области, ограниченной окружностью с радиусом, равным радиусу зеркала телескопа, а в пространстве углов внутри области, ограниченной окружностью с угловым радиусом $10.8^{\prime}$. Данная величина соответствует найденному угловому размеру пикселя системы зеркала телескопа и одиночного конуса.

Детекторы располагались на расстоянии $1 \mathrm{~mm}$ от выходного окна конуса, что позволило минимизировать потери сигнала.

Вследствие неидеальности конуса, а именно наличия полигональной структуры, часть фотонов не проходила через него и отражалась в обратном направлении. Данные фотоны регистрировались на плоскости 2 (рис. 3),

Таблица 2. Результаты численного моделирования по определению трансмиссии конусов Уинстона

\begin{tabular}{c|c|c|c}
\hline Параметр & $\theta=26.56^{\circ}$ & $\theta=30.00^{\circ}$ & $\theta=35.00^{\circ}$ \\
\hline $\begin{array}{c}\text { Фотоны, отраженные } \\
\text { в обратную сторону, \% }\end{array}$ & 7.20 & 1.45 & 0.06 \\
\hline $\begin{array}{c}\text { Потери при } \\
\text { отражении, \% }\end{array}$ & 9.92 & 7.43 & 5.97 \\
\hline $\begin{array}{c}\text { Потери на } \\
\text { детекторах, \% }\end{array}$ & 13.49 & 17.53 & 29.38 \\
\hline $\begin{array}{c}\text { Трансмиссия } \\
\text { конуса, \% }\end{array}$ & 69.38 & 72.58 & 64.43
\end{tabular}



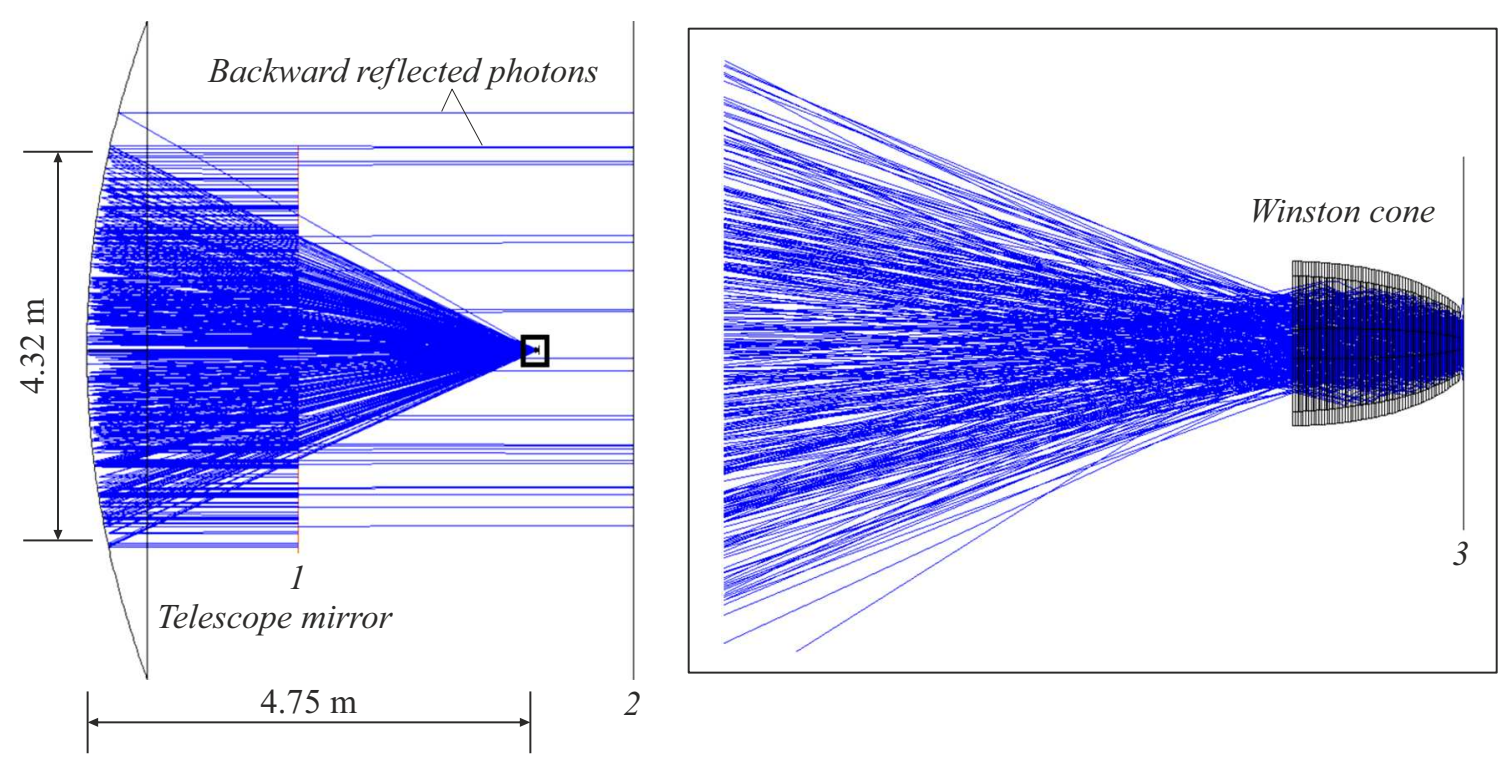

Рис. 3. Численная модель в пакете Zетаx, включающая зеркало телескопа и одиночный конус Уинстона: 1 - плоскость старта фотонов, 2 - плоскость регистрации фотонов, отраженных в обратную сторону, 3 - плоскость детекторов.

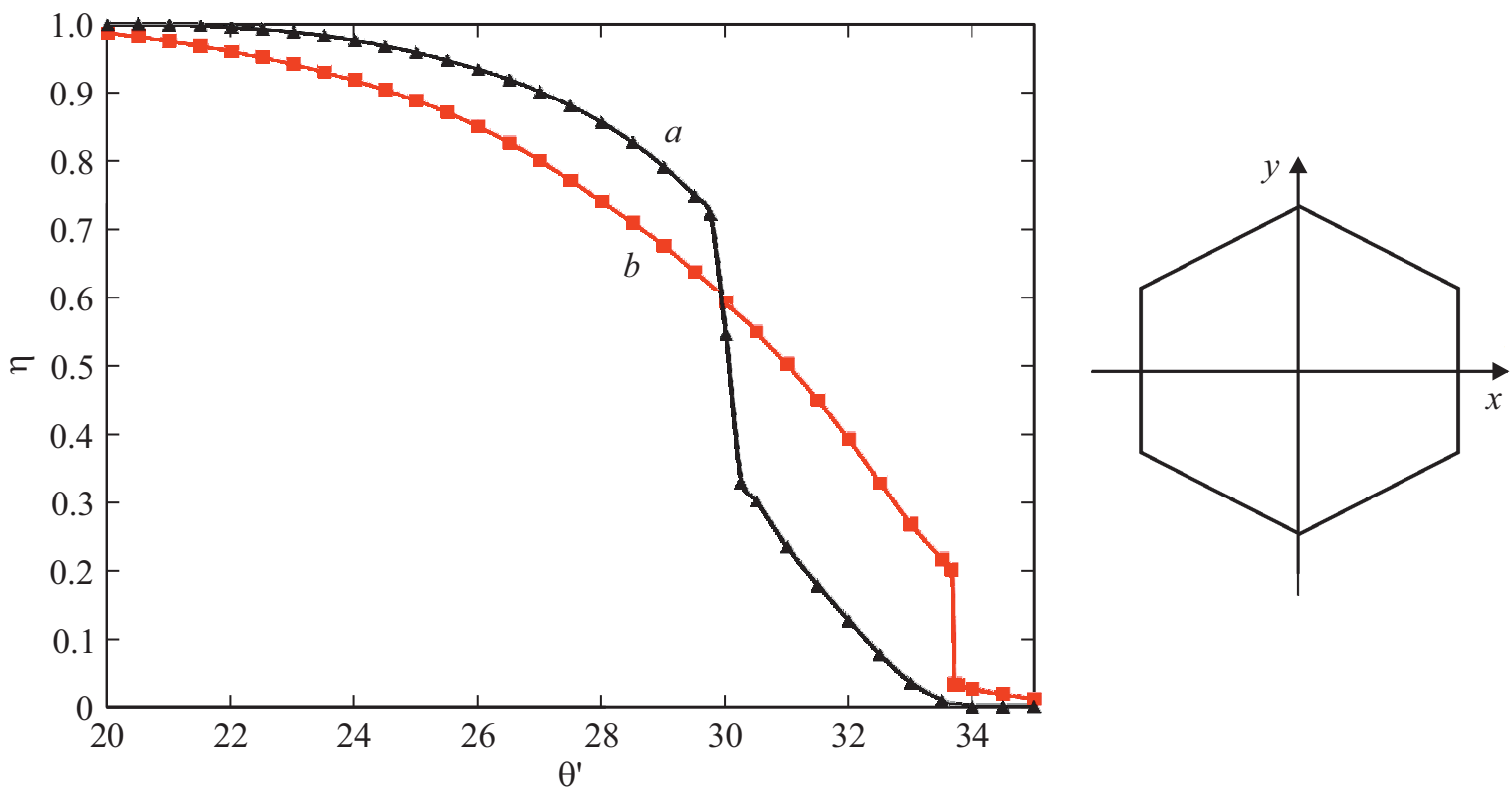

Рис. 4. Результаты численного расчета функции пропускания $\eta$ шестиугольного конуса Уинстона при фиксированном угле наклона траектории фотонов к оси конуса $\theta^{\prime}: a-$ по отношению к оси $x, b-$ по отношению к оси $y$.

что позволило определить потери сигнала, вызванные неидеальностью конуса.

Результаты расчета трансмиссии для 3 вариантов конусов приведены в табл. 2. Наиболее существенной причиной снижения сигнала оказались потери фотонов в области детекторов. Связаны они, прежде всего, с несоответствием формы используемых детекторов и выходного окна конуса, таким образом не все фотоны, прошедшие через конус, попадают на поверхность детектора. В целом можно отметить, что вследствии законов сохранения совокупный сигнал фотонов, приходящий на детекторы, для конуса Уинстона в идеальном случае зависит только от их площади и не зависит от конструкции конусов. Однако данное утверждение справедливо только для идеального случая, когда коэффициент отражения равен единице, а форма детектора совпадает с формой выходного окна конуса.

Потери при отражении от внутренних поверхностей конусов составили не более $10 \%$. В то же время для конструкции конуса с углом 26.56 градусов (при таком угле радиус описанной окружности выходного шестиугольника в 2 раза меньше радиуса вписанной окружно- 

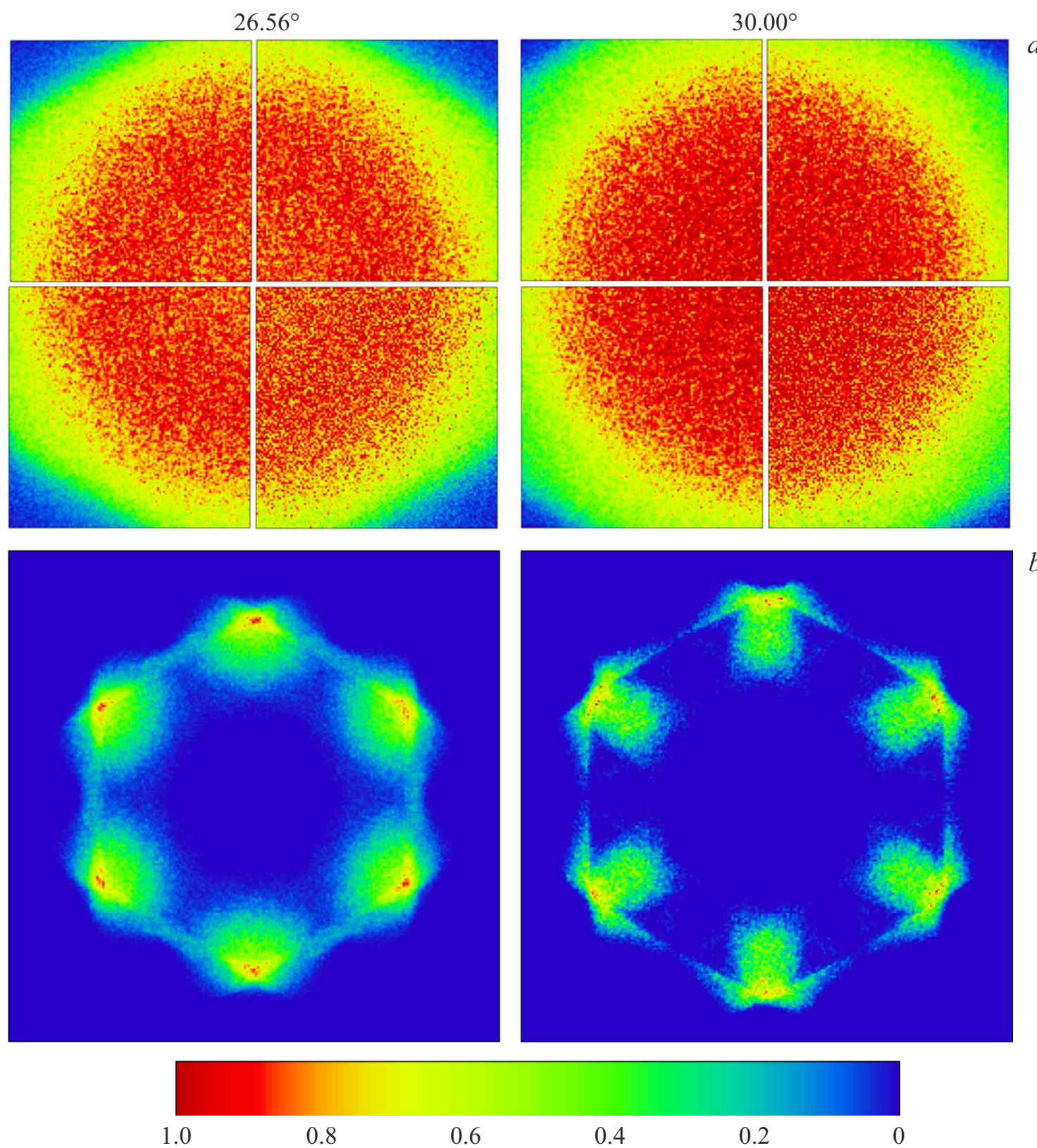

Рис. 5. Распределение нормированной интенсивности потока фотонов: $a-$ в плоскости детекторов, $b-$ в плоскости регистрации фотонов, отраженных в обратную сторону.

сти входного шестиугольника) заметные потери потока фотонов были связаны с их отражением в обратную сторону.

Расчет распределения нормированной интенсивности потока фотонов в плоскости детекторов (рис. 5, a) показал, что, несмотря на шестиугольную форму конусов, данное распределение в целом симметрично относительно оси конуса. Распределение по интенсивности фотонов, отраженных в обратном направлении в плоскости 2, приведено на рис. 5, $b$. Из рисунка видно, что именно отражения в области углов полигональной структуры конуса вносят основной вклад в уменьшение интенсивности регистрируемых фотонов.

\section{Выводы}

Количественное моделирование системы оптических концентраторов на основе шестиугольных конусов Уинстона для черенковского гамма-телескопа показало, что из 3 рассмотренных вариантов конус с углом обзора 30 градусов обладает наилучшими характеристиками при использовании заданной конфигурации из 4 детекторов. По результатам численного моделирования были определены потери при отражении от внутренних поверхностей конуса, они составили $7.43 \%$, потери, вызванные несоответствием выходного окна конуса и детекторов, составили $17.53 \%$. Таким образом, найден- 
ная численно трансмиссия данного конуса составила более $72 \%$.

Было проведено сравнение результатов, полученных для рассматриваемой системы, с опубликованными данными. В частности, в работе [20] приведены результаты численного моделирования для различных конфигураций конуса Уинстона, отличающихся формой входного и выходного окна. По результатам сравнения можно сделать вывод, что рассматриваемая конструкция имеет наиболее быстрый спад функции пропускания, и соответственно наиболее высокую эффективность подавления шумового фона фотонов. По данному параметру рассматриваемая конструкция практически не уступает параболическому конусу Уинстона.

\section{Финансирование работы}

Авторы благодарят Российский научный фонд (РНФ) за поддержку в рамках проекта 19-72-20045.

\section{Конфликт интересов}

Авторы заявляют, что у них нет конфликта интересов.

\section{Список литературы}

[1] Y. Becherini, A. Djannati-Atai, M. Punch, K. Bernlöhr, S. Ehlert, J. Masbou, E. Moulin, M. Paz Arribas, H.E.S.S. Collaboration. AIP Conf. Proceed., 1085, 738 (2008). DOI: $10.1063 / 1.3076784$

[2] S. Ansoldi, L.A. Antonelli, P. Antoranz, A. Babic, P. Bangale, U. Barres de Almeida, J.A. Barrio, J. Becerra González, W. Bednarek, E. Bernardini, B. Biasuzzi, A. Biland, O. Blanch, S. Bonnefoy, G. Bonnoli, F. Borracci, T. Bretz, E. Carmona, A. Carosi, P. Colin, E. Colombo, J.L. Contreras, J. Cortina, S. Covino, P. Da Vela, F. Dazzi, A. De Angelis, G. De Caneva, B. De Lotto, E. de Oña Wilhelmi, C. Delgado Mendez, F. Di Pierro, D. Dominis Prester, D. Dorner, M. Doro, S. Einecke, D. Eisenacher Glawion, D. Elsaesser, A. Fernández-Barral, D. Fidalgo, M.V. Fonseca, L. Font, K. Frantzen, C. Fruck, D. Galindo, R.J. García López, M. Garczarczyk, D. Garrido Terrats, M. Gaug, N. Godinović, A. González Muñoz, S.R. Gozzini, Y. Hanabata, M. Hayashida, J. Herrera, K. Hirotani, J. Hose, D. Hrupec, G. Hughes, W. Idec, H. Kellermann, M.L. Knoetig, K. Kodani, Y. Konno, J. Krause, H. Kubo, J. Kushida, A. La Barbera, D. Lelas, N. Lewandowska, E. Lindfors, S. Lombardi, F. Longo, M. López, R. López-Coto, A. López-Oramas, E. Lorenz, M. Makariev, K. Mallot, G. Maneva, K. Mannheim, L. Maraschi, B. Marcote, M. Mariotti, M. Martínez, D. Mazin, U. Menzel, J.M. Miranda, R. Mirzoyan, A. Moralejo, P. Munar-Adrover, D. Nakajima, V. Neustroev, A. Niedzwiecki, M. Nevas Rosillo, K. Nilsson, K. Nishijima, K. Noda, R. Orito, A. Overkemping, S. Paiano, M. Palatiello, D. Paneque, R. Paoletti, J.M. Paredes, X. Paredes-Fortuny, M. Persic, J. Poutanen, P.G. Prada Moroni, E. Prandini, I. Puljak, R. Reinthal, W. Rhode, M. Ribó, J. Rico, J. Rodriguez Garcia, T. Saito, K. Saito, K. Satalecka,
V. Scalzotto, V. Scapin, C. Schultz, T. Schweizer, S.N. Shore, A. Sillanpää, J. Sitarek, I. Snidaric, D. Sobczynska, A. Stamerra, T. Steinbring, M. Strzys, L. Takalo, H. Takami, F. Tavecchio, P. Temnikov, T. Terzić, D. Tescaro, M. Teshima, J. Thaele, D.F. Torres, T. Toyama, A. Treves, J. Ward, M. Will, R. Zanin. A\&A, 585, A133 (2016). DOI: $10.1051 / 0004-6361 / 201526853$

[3] U. Barres de Almeida. Astronomische Nachrichten, $336(8-9), 795$ (2015).

[4] A.M. Bykov, F.A. Aharonian, A.M. Krassilchtchikov, E.E. Kholupenko, P.N. Aruev, D.A. Baiko, A.A. Bogdanov, G.I. Vasilyev, V.V. Zabrodskii, S.V. Troitsky, Yu.V. Tuboltsev, A.A. Kozhberov, K.P. Levenfish, Yu.V. Chichagov. Journal of Technical Physics, 62 (6), 819 (2017).

[5] J. Aleksi' c, S. Ansoldi, L.A. Antonelli, P. Antoranz, A. Babic, P. Bangale, M. Barceló, J.A. Barrio, J. BecerraGonzález, W. Bednarek, E. Bernardini, B. Biasuzzi, A. Biland, M. Bitossi, O. Blanch, S. Bonnefoy, G. Bonnoli, F. Borracci, T. Bretz, E. Carmona, A. Carosi, R. Cecchi, P. Colin, E. Colombo, J.L. Contreras, D. Corti, J. Cortina, S. Covino, P. DaVela, F. Dazzi, A. De Angelis, G. DeCaneva, B. De Lotto, E. de Oña Wilhelmi, C. Delgado Mendez, A. Dettlaff, D. Dominis Prester, D. Dorner, M. Doro, S. Einecke, D. Eisenacher, D. Elsaesser, D. Fidalgo, D. Fink, M.V. Fonseca, L. Font, K. Frantzen, C. Fruck, D. Galindo, R.J. García López, M. Garczarczyk, D. Garrido Terrats, M. Gaug, G. Giavitto, N. Godinovi' c, A. González Muñoz, S.R. Gozzini, W. Haberer, D. Hadasch, Y. Hanabata, M. Hayashida, J. Herrera, D. Hildebrand, J. Hose, D. Hrupec, W. Idec, J.M. Illa, V. Kadenius, H. Kellermann, M.L. Knoetig, K. Kodani, Y. Konno, J. Krause, H. Kubo, J. Kushida, A. La Barbera, D.Lelas, J.L. Lemus, N. Lewandowska, E. Lindfors, S. Lombardi, F. Longo, M. López, R. López-Coto, A. López-Oramas, A. Lorca, E. Lorenz, I. Lozano, M. Makariev, K. Mallot, G. Maneva, N. Mankuzhiyil, K. Mannheim, L. Maraschi, B. Marcote, M. Mariotti, M. Martínez, D. Mazin, U.Menzel, J.M. Miranda, R. Mirzoyan, A. Moralejo, P. Munar-Adrover, D. Nakajima, M. Negrello, V. Neustroev, A. Niedzwiecki, K. Nilsson, K. Nishijima, K. Noda, R. Orito, A. Overkemping, S. Paiano, M. Palatiello, D. Paneque, R. Paoletti, J.M. Paredes, X. Paredes-Fortuny, M. Persic, J. Poutanen, P.G. PradaMoroni, E. Prandini, I. Puljak, R. Reinthal, W. Rhode, M. Ribó, J. Rico, J. Rodriguez Garcia, S. Rügamer, T. Saito, K. Saito, K. Satalecka, V.Scalzotto, V. Scapin, C. Schultz, J. Schlammer, S. Schmidl, T. Schweizer, S.N. Shore, A. Sillanpää, J. Sitarek, I. Snidaric, D. Sobczynska, F. Spanier, A. Stamerra, T. Steinbring, J. Storz, M. Strzys, L. Takalo, H. Takami, F. Tavecchio, L.A. Tejedor, P. Temnikov, T. Terzi 'c, D. Tescaro, M. Teshima, J. Thaele, O. Tibolla, D.F. Torres, T. Toyama, A. Treves, P. Vogler, H. Wetteskind, M. Will, R. Zanin. Astroparticle Physi., 72, 61 (2016).

[6] G. Giavitto, T. Ashton, A. Balzer, D. Berge, F. Brun, Th. Chaminade, E. Delagnes, G. Fontaine, M. Füßling, B. Giebels, J.-F. Glicenstein, T. Gräber, J. Hinton, A. Jahnke, S. Klepser, M. Kossatz, A. Kretzschmann, V. Lefranc, H. Leich, H. Lüdecke, I. Lupova, P. Manigot, V. Marandon, E. Moulin, M. de Naurois, P. Nayman, S. Ohm, M. Penno, D. Ross, D. Salek, M. Schade, Th. Schwab, R. Simoni, C. Stegmann, C. Steppa, J. Thornhill, F. Toussnel. Nucl. Instruments Methods in Phys. Res. Section A, 876, 35 (2017). DOI: 10.1016/j.nima.2016.12.057 
[7] L.A. Kuzmichev, I.I. Astapov, P.A. Bezyazeekov, V. Boreyko, A.N. Borodin, N.M. Budnev, R. Wischnewski, A.Y. Garmash, A.R. Gafarov, N.V. Gorbunov, V.M. Grebenyuk, O.A. Gress, T.I. Gress, A.A. Grinyuk, O.G. Grishin, A.N. Dyachok, A.V. Zagorodnikov, V.L. Zurbanov, A.L. Ivanova, Y.A. Kazarina, N.N. Kalmykov, N.I. Karpov, V.V. Kindin, P.S. Kirilenko, S.N. Kiryuhin, V.A. Kozhin, R.P. Kokoulin, K.G. Kompaniets, E.E. Korosteleva, E.A. Kravchenko, M. Kunnas, A. Chiavassa, A.A. Lagutin, V.V. Lenok, B.K. Lubsandorzhiev, N.B. Lubsandorzhiev, R.R. Mirgazov, R. Mirzoyan, R.D. Monkhoev, R. Nachtigall, E.A. Osipova, M.I. Panasyuk, L.V. Pankov, A.L. Pakhorukov, A.A. Petrukhin, V.A. Poleschuk, M. Popesku, E.G. Popova, A. Porelli, E.B. Postnikov, V.V. Prosin, V.S. Ptuskin, A.A. Pushnin, R.I. Raikin, G.I. Rubtsov, Ya.I. Sagan, V.S. Samoliga, L.G. Sveshnikova, Yu.A. Semeney, A.Y. Sidorenkov, A.A. Silaev, A.V. Skurikhin, M. Slunecka, A.V. Sokolov, V.P. Sulakov, V.A. Tabolenko, B.A. Tarashansky, L.G. Tkachev, A.V. Tkachenko, M. Tluczykont, O.L. Fedorov, A.D. Horns, C. Spiering, A.Sh.M. Elshoukrofy, I.I. Yashin. Phys. Atom. Nuclei, 81 (4), 497 (2018).

[8] N. Lubsandorzhiev, I. Astapov, P. Bezyazeekov, V. Boreyko, A. Borodin, M. Brueckner, N. Budnev, A. Chiavassa, A.N. Dyachok, O. Fedorov, A. Gafarov, A. Garmash, N. Gorbunov, V. Grebenyuk, O. Gress, T. Gress, O. Grishin, A. Grinyuk, D. Horns, A. Igoshin, A. Ivanova, N. Kalmykov, Y. Kazarina, V. Kindin, P. Kirilenko, S. Kiryuhin, R. Kokoulin, K. Kompaniets, E. Korosteleva, V.V. Kozhin, E. Kravchenko, M. Kunnas, L. Kuzmichev, Y. Lemeshev, V. Lenok, B. Lubsandorzhiev, R. Mirgazov, R. Mirzoyan, R. Monkhoev, R. Nachtigall, E. Osipova, A. Pakhorukov, M.I. Panasyuk, L. Pankov, A. Petrukhin, V. Poleschuk, E. Popescu, E. Popova, A. Porelli, E. Postnikov, V. Prosin, V. Ptuskin, E.V. Rjabov, G. Rubtsov, A. Pushnin, Y. Sagan, B. Sabirov, V. Samoliga, Y. Semeney, A. Silaev, A. Silaev(junior), A. Sidorenkov, A.V. Skurihin, V. Slunecka, A.V. Sokolov, C. Spiering, L. Sveshnikova, V. Tabolenko, B. Tarashansky, A. Tkachenko, L. Tkachev, M. Tluczykont, R. Wischnewski, A. Zagorodnikov, D. Zhurov, V. Zurbanov, I. Yashin. In 35th Intern. Cosmic Ray Conf. (ICRC2017). Intern. Cosmic Ray Conf., 301 (1), 757 (2017).

[9] A.A. Bogdanov, E.E. Kholupenko, Yu.V. Tuboltsev, Yu.V. Chichagov, Latvian J. Phis. Tech. Sci., 1, 13 (2020). DOI: $10.2478 / \mathrm{lpts}-2020-0002$

[10] Е.Е. Холупенко, А.М. Красильщиков, Д.В. Бадмаев, А.А. Богданов, Ю.В. Тубольцев, Ю.В. Чичагов, А.С. Антонов, Д.О. Кулешов, Е.М. Хилькевич. ЖТФ, 90 (6), 954 (2020). DOI: 10.21883/JTF.2020.06.49278.354-19 [E.E. Kholupenko, A.M. Krassilchtchikov, D.V. Badmaev, A.A. Bogdanov, Yu.V. Tuboltsev, Yu.V. Chichagov, A.S. Antonov, D.O. Kuleshov, E.M. Khil'kevich. Tech. Phys., 65 (6), 886 (2020). DOI: 10.1134/S1063784220060158]

[11] А.А. Богданов, Ю.В. Тубольцев, Ю.В. Чичагов, Е.Е. Холупенко, А.М. Красильщиков. ЖТФ, 5, 821 (2021). DOI: $10.21883 /$ JTF.2021.05.50695.270-20

[12] F.A. Aharonian, A.G. Akhperjanian, A.S. Kankanian, R.G. Mirzoyan, A.A. Stepanian, N. Müller, M. Samorski, W. Stamm, M. Bott-Bodenhausen, E.A. Lorenz, P. Sawallisch. Proceedings of the 22nd International Cosmic Ray Conference. 11-23 August, 1991. Dublin, Ireland. Under the auspices of the International Union of Pure and Applied Physics (IUPAP), Volume 2, Contributed Papers, OG Sessions 6-11. Dublin: The Institute for Advanced Studies, 1991, p. 615
[13] J. Bolmont, P. Corona, P. Gauron, P. Ghislain, C. Goffin, L. Guevara Riveros, J.-F. Huppert, O. Martineau-Huynh, P. Nayman, J.-M. Parraud, J.-P. Tavernet, F. Toussenel, D. Vincent, P. Vincent, W. Bertoli, P. Espigat, M. Punch, D. Besin, E. Delagnes, J.-F. Glicenstein, Y. Moudden, P. Venault, H. Zaghia, L. Brunetti, P.-Y. David, J-M. Dubois, A. Fiasson, N. Geffroy, I. Gomes Monteiro, L. Journet, F. Krayzel, G. Lamanna, T. Le Flour, S. Lees, B. Lieunard, G. Maurin, P. Mugnier, J.-L. Panazol, J. Prast, L.-M. Chounet, B. Degrange, E. Edy, G. Fontaine, B. Giebels, S. Hormigos, B. Khélifi, P. Manigot, P. Maritaz, M. de Naurois, M. Compin, F. Feinstein, D. Fernandez, J. Mehault, S. Rivoire, S. Royer, M. Sanguillon, G. Vasileiadis. Instrumentation Methods for Astrophys., Res. Sec. A, 761, 46 (2014).

[14] J.A. Aguilar, A. Basili, V. Boccone, F. Cadoux, A. Christov, D. della Volpe, T. Montaruli, Ł. Płatos, M. Rameez. Astroparticle Phys., 60, 32 (2015). DOI: 10.1016/j.astropartphys.2014.05.010

[15] F. Hénault, P.-O. Petrucci, L. Jocou, B. Khélifi, P. Manigot, S. Hormigos, J. Knödlseder, J.-F. Olive, P. Jean, M. Punch. Proc. SPIE 8834, Nonimaging Optics: Efficient Design for Illumination and Solar Concentration X, 883405 (18 September 2013) DOI: 10.1117/12.2024049

[16] XP1911, product specification, http://www.hzcphotonics.com/products/XP1911.pdf, http://www.isuzuoptics.com.tw/data/product/photonis/pmt/ 19mm-1.pdf

[17] Электронный ресурс. Режим доступа: http://www.zemax.com

[18] R. Winston, L. Jiang, M. Ricketts. Adv. Opt. Photon., 10 (2), 484 (2013).

[19] T. Coopera, F. Dählera, G. Ambrosetti, A. Pedretti. Solar Energy, 95, 308 (2013).

[20] J.P. Koschinsky. Development of a 61-Pixel Camera for the IceAct Imaging Air Cherenkov Telescope, 2017, Master's thesis, RWTH Aachen University. 